\title{
Author Correction: Antigen-capturing nanoparticles improve the abscopal effect and cancer immunotherapy
}

Yuanzeng Min, Kyle C. Roche, Shaomin Tian, Michael J. Eblan, Karen P. McKinnon, Joseph M. Caster, Shengjie Chai, Laura E. Herring, Longzhen Zhang, Tian Zhang, Joseph M. DeSimone, Joel E. Tepper, Benjamin G. Vincent, Jonathan S. Serody and Andrew Z. Wang

Correction to: Nature Nanotechnology https://doi.org/10.1038/nnano.2017.113, published online 26 June 2017.

For this Letter, we would like to make the readers aware that in the left panel of Fig. 4a the lymph nodes from the different experimental groups were imaged in separate 96-well plates using the same parameters, thus each column in the figure should have been more clearly separated. In addition, the colour scale bar was missing. The corrected version of Fig. 4a is shown below.

Furthermore, in the section 'Uptake of AC-NPs by APCs in irradiated tumours and lymph nodes' of the Methods, more experimental details and the imaging parameters should have been included. The sentence "Rhodamine B-labelled AC-NPs within lymph nodes were imaged with an IVIS imaging system" should have read "Rhodamine B-labelled AC-NPs within lymph nodes were imaged with an IVIS imaging system (exposure time: $0.5 \mathrm{~s}$; excitation: $535 \mathrm{~nm}$; emission filter: DsRed, which has the same excitation wavelength and emission wavelength as the rhodamine B dye. No automated scaling)." The following text should have run on: "96-well plates were used for the background, which was automatically subtracted from each new sample. Five lymph nodes from the same experimental group were put in plate A and imaged simultaneously. Following transfer into plate B on ice, the five lymph nodes from the subsequent experimental group were measured in plate A, and so on. Uptake of AC-NPs into APCs was assessed by flow cytometric analysis of single-cell suspensions. These were stained with anti-mouse CD11c, F4/80 and B220 (Supplementary Table 1).”

In the Supplementary Information, in the left panel of Supplementary Fig. 8a each column should have been more clearly separated, and

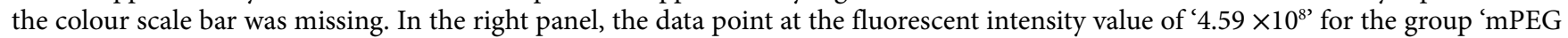
AC-NPs ( $1 \mathrm{~h}$ )' was incorrect, and should have been at ' $4.59 \times 10^{7}$ '. The associated error bar has also been amended accordingly. The corrected version of Supplementary Fig. 8 a is shown below. Moreover, Supplementary Fig. 16 was missing, which shows the original images that correspond to the cropped images shown in Fig. 4a and Supplementary Fig. 8a. It is shown below with its caption.

These errors do not affect the conclusions of the paper. The original Letter has not been corrected.
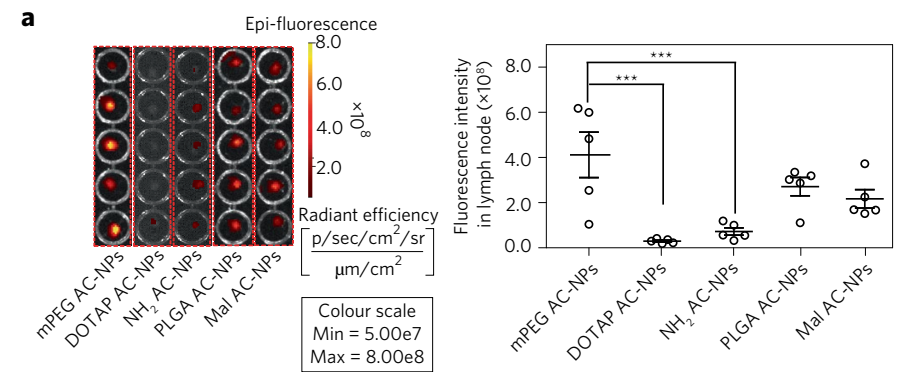

Fig. 4 | Corrected. 
a
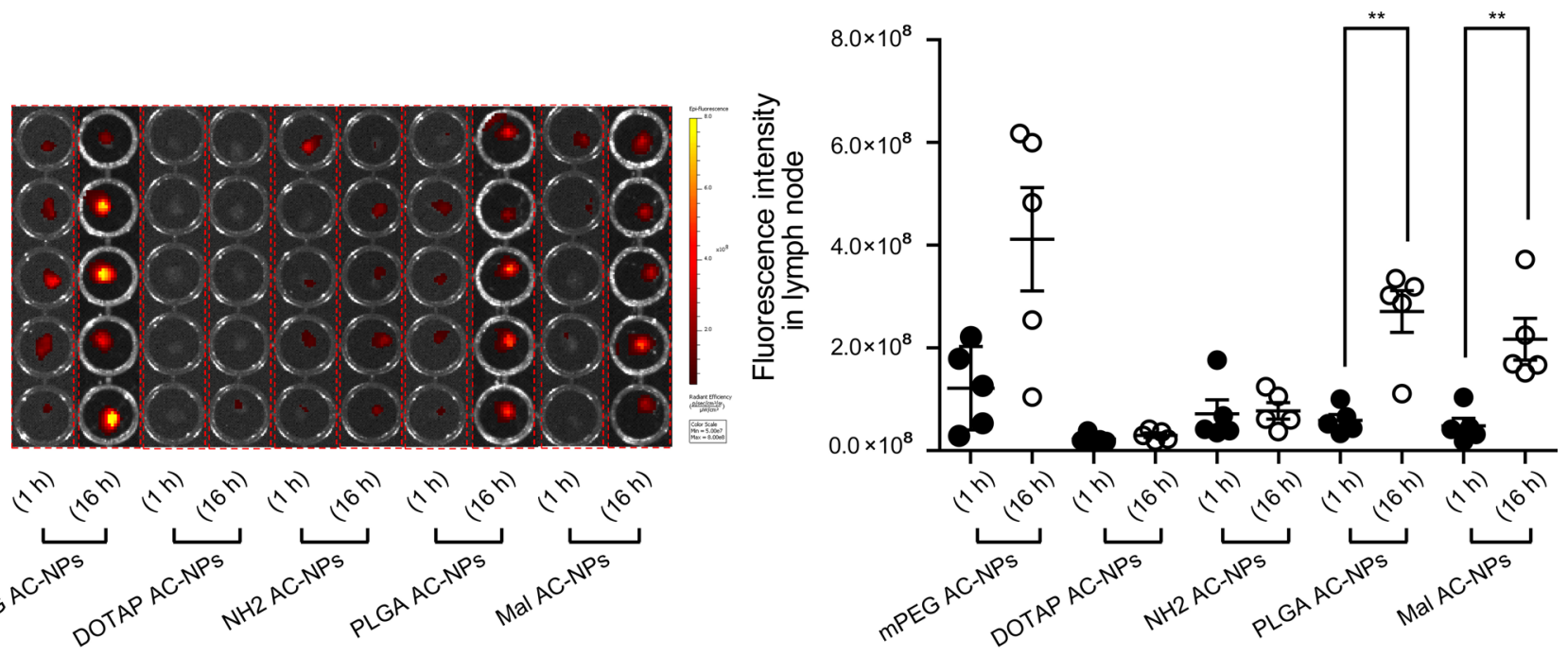

Supplementary Fig. 8 | Corrected.
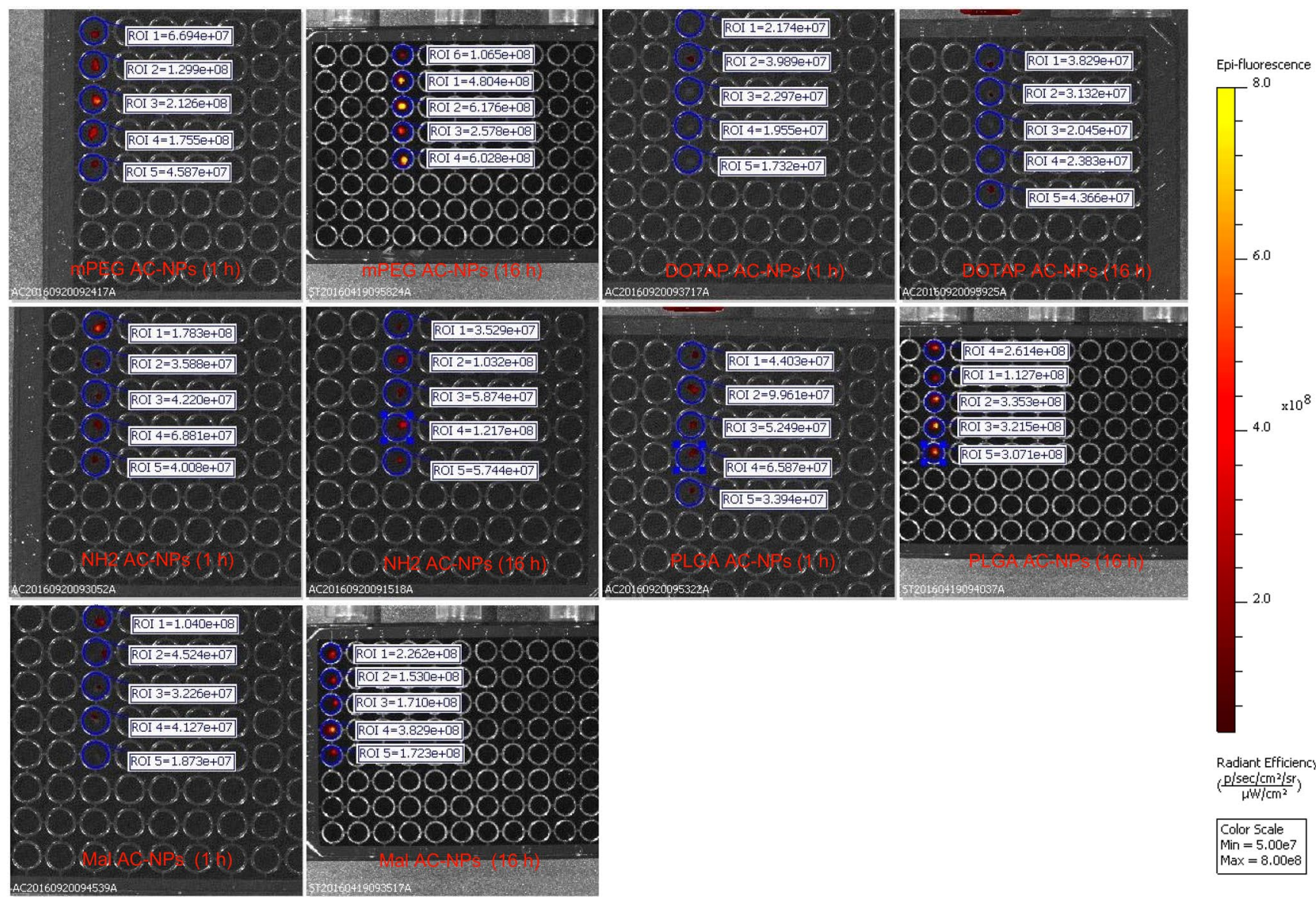

Radiant Efficiency

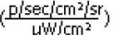

Color Scale

Supplementary Fig. 16 | The original images corresponding to Fig. 4a and Supplementary Fig. 8a. Images of lymph nodes at 1 and 16 hours after intratumoural injection of fluorescently-labeled AC-NPs $(n=5)$.

Published online: 12 February 2021

https://doi.org/10.1038/s41565-021-00864-w

(C) The Author(s), under exclusive licence to Springer Nature Limited 2021 